\title{
Effect of Household Composition on Young Adult Survey Participation
}

Robin Gentry, Barbara C O'Hare ${ }^{\dagger}$

Tags: survey practice

\section{Survey Practice}

Vol. 1, Issue 4, 2008

Effect of Household Composition on Young Adult Survey Participation

To successfully complete an interview in a telephone survey, we need the person who answers the phone to cooperate and provide access to the intended respondent or respondents within the household. The first contact is a household contact, often a screening call. The contact person acts as a gatekeeper to other household members, and may influence the likelihood of cooperation among these other members. Characteristics of the household are known to influence the likelihood of contact and response ${ }^{1}$. This article discusses the importance of household composition in survey cooperation. In particular, it focuses on the young adults who often respond at rates lower than their older counterparts.

The number and the age and sex of persons living in the household, in this study conducted by Arbitron, Inc. were found to be related to survey cooperation rates. If the contact person is enthusiastic about participating in the survey, he or she may be more effective in encouraging other household members to do the same. Alternatively, if the contact person fails to cooperate with the request or is negative about the request, other household members' participation may be jeopardized. Thus, there may be a complex set of household composition factors which drive non-response in an RDD survey design.

Household composition effects may play a particularly important role for young adults between the ages of 18 and 24 . There is a great deal of literature which suggests that monetary incentives are an effective method of increasing response rates ${ }^{2}$. Furthermore, the leverage-saliency theory posits that survey response is dependent on how salient each positive and negative aspect of the survey task is made during the survey request and how much leverage each of those aspects has on an individual's response propensity ${ }^{3}$. Based on this

\footnotetext{
* Institution: Arbitron, Inc.

† Institution: Arbitron, Inc.

1 Groves, R. and M. Couper. 1998. Nonresponse in household interview surveys. John Wiley, New York.

2 Singer, E. 2002. The use of incentives to reduce nonresponse in household surveys. In: (R.M. Groves, D.A. Dillman, J.L. Eltingem and R.J.A. Little, eds.). Survey nonresponse. Wiley, New York. pp. 163-177.

3 Groves, R., E. Singer and A.D. Corning. 1999. Leverage-saliency theory of survey participation: description and an illustration. Public Opinion
} 
theory, incentives may be used in an attempt to overcome the negative leverage associated with the burden of completing the survey.

However this approach is not fully informed because it ignores the potential effect of household composition. In a 2004 follow-up survey ${ }^{4}$ conducted with 18 to 24 year old men whose households participated in the Arbitron survey, nearly 60 percent of the respondents reported that another household member took a particular interest in making sure they completed their survey diaries. The most common person to fill this "shepherd" role was their mother (56.6\%), followed by their father $(15.8 \%)$ or their spouse $(11.8 \%)$.

Another Arbitron experiment of a targeted mailing to men 18 to 24 years old, found that when all household members were under the age of 25 the young men in the household were significantly less likely to return their radio listening diaries than when the household contained at least one person outside that age range (a return rate of $42.1 \%$ in younger households vs. $56.7 \%$ in older households).

\section{STUDY DESIGN}

The most recent Arbitron study of the effects of household composition on survey return of adults ages 18 to 24 takes advantage of new data from enumeration of all household members 12 and older by age and sex. The Arbitron survey of radio listening starts with an RDD phone interview of any adult in the household from whom consent is obtained to send one-week diaries for all persons 12 or older to record their radio listening. In this study, we analyzed the enumeration data of 50,904 households from 91 metropolitan areas representative of differing population sizes, demographic composition and geographic regions from our Winter 2008 survey.

The logistic regression analyses were conducted in two stages, first examining the predictive value of different household characteristics on the likelihood of survey return for young adults 18 to 24 years of age. Our second stage of analysis grouped the young adult households into types based on their composition (e.g. only young adults, young adults living with someone 35+, couples, etc.). The first model allows us to look at household characteristics which play the largest role in young adult diary return while the second set allows us to contrast the probability of survey return across different types of households. The dependent variable is whether or not all of the household members in the target demographic (men or women ages 18-24) returned a diary.

Household characteristics were selected based on prior knowledge of key correlates of diary return and included the number of men 18-24 or number 
of women 18-24, household size, presence of person age 35 or older, and the age and sex of the person who completed the phone interview. The models controlled for other variables related to survey return, including other sample characteristics and survey attributes, such as incentives. Because these models explore the characteristics related to household interactions and composition, they were run only for households with two or more respondents. Detailed descriptions of the variables and models are available from the authors.

\section{RESULTS}

Key findings, as seen in Table 1, on the household characteristics that predict young adult diary return include:

Table 1 Odds Ratios of Household Composition Predictors on Likelihood of All Diaries Returned by Men and Women Ages 18-24.

\begin{tabular}{|c|c|c|}
\hline & $\begin{array}{l}\text { Young Men 18-24 } \\
(\mathrm{N}=4608)\end{array}$ & $\begin{array}{l}\text { Young Women 18-24 } \\
(\mathrm{N}=4540)\end{array}$ \\
\hline & Odds Ratio & Odds Ratio \\
\hline \multicolumn{3}{|l|}{ Household Composition } \\
\hline \multicolumn{3}{|l|}{$\mathrm{PPH} 2$} \\
\hline $\mathrm{PPH} 3$ & $0.613^{*}$ & $0.675^{*}$ \\
\hline $\mathrm{PPH} 4$ & $0.547^{*}$ & $0.728^{*}$ \\
\hline PPH 5+ & $0.468^{*}$ & $0.637^{*}$ \\
\hline Female Consenter & $1.213^{*}$ & $1.537^{*}$ \\
\hline Consenter Age & $1.040^{*}$ & $1.052^{*}$ \\
\hline Consenter Age2 & $1.000^{*}$ & $1.000^{*}$ \\
\hline No Adult Female & $0.511^{*}$ & NA \\
\hline HH all Under Age 35 & $0.762^{*}$ & $0.760^{*}$ \\
\hline Number of People in Target Group & $0.586^{*}$ & $0.578^{*}$ \\
\hline Constant & 1.353 & 0.456 \\
\hline Statistically significant at $95 \%$ confidence lev & & \\
\hline
\end{tabular}

Regardless of age or gender, the larger the household the lower the odds of receiving diaries from all adults ages 18-24 in the household.

A household with all adults under the age of 35 is associated with lower odds of diary return for Males 18-24 and Females 18-24.

The presence of an adult female, age 18 or older, in a young male household improves the odds of diary return.

If the person who agrees on behalf of the household to participate in the survey is female and/or older, the odds of diary return increase significantly.

As the number of young adults in the target group increases, the likelihood of getting diaries back from each of them decreases. 
The second set of models used the findings from the household characteristics models to define three main groups - young adult couples, other combinations of young adults living together, and young adults living in mixed ages households. We were interested in the effect of these household "types" on the probability of receiving all diaries back from the young men or young women. Findings from these models (Table 2) include:

Table 2 Odds Ratios of Household Type Predictors on Likelihood of All Diaries Returned from Men and Women Ages 18-24.

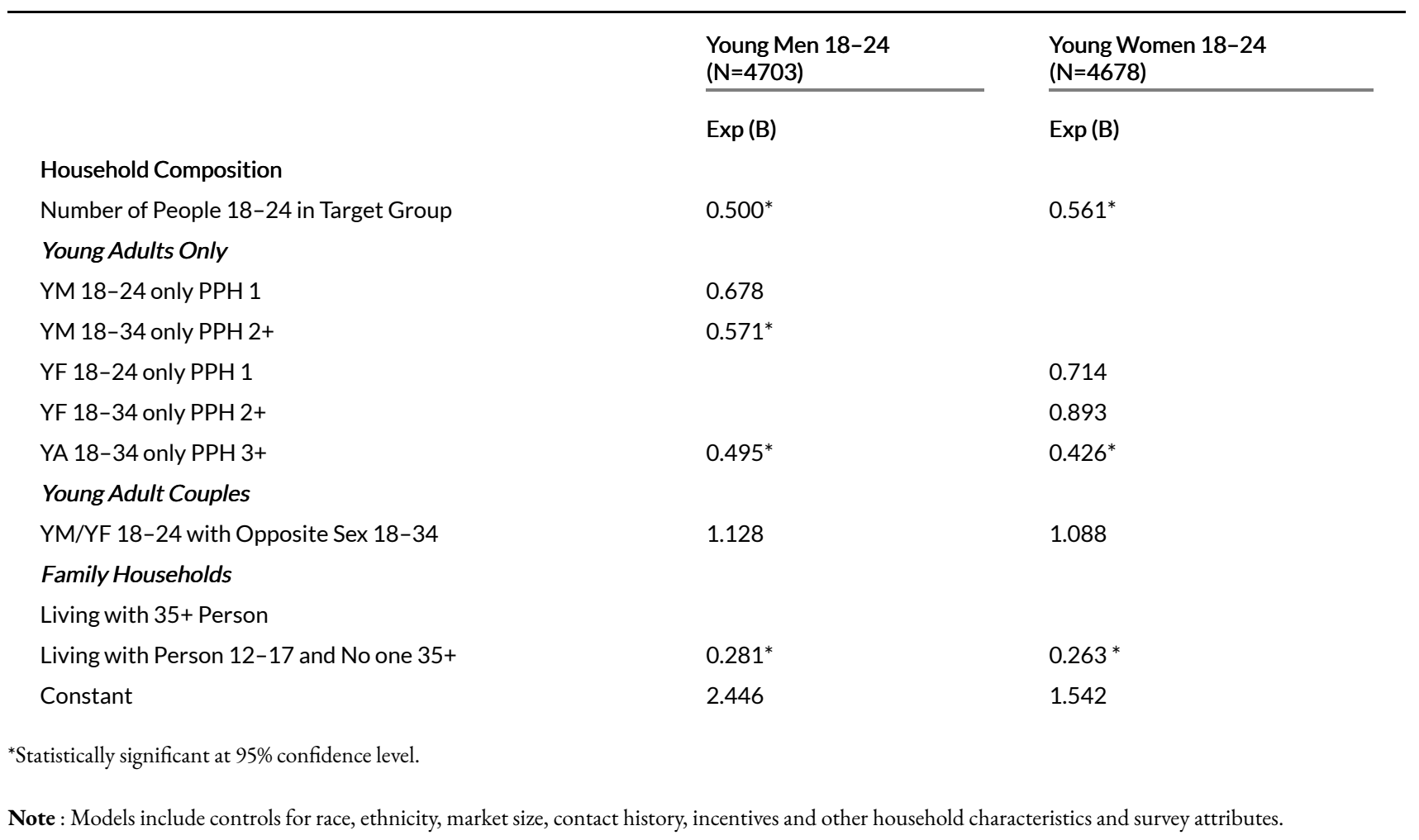

In comparison to a household with a member age 35 or older, a group of 3 or more young adults living together are less than half as likely to return all the diaries from the 18-24 year olds.

Young couples' return rates are comparable to those of 18-24 year olds living with older adults.

Men 18-24 living with only other men 18-34 return all their diaries at a $40 \%$ lower rate (odds ratio $=0.571, \mathrm{p}<0.05$ ) than their counterparts living with someone age 35 or more. This is not the case for women 18-24 (odds ratio $=0.893$, n.s.)

\section{CONCLUSIONS}

Who the respondent lives with can make a difference in the likelihood of participating in the survey. The household characteristic model indicates that odds of survey return decline with household size, but are increased with the presence of a woman, the presence of someone age 35 or older, and an older 
and/or female consenter. In 18-24 year old male households, the odds of survey return decline sharply if they are living with only other young men or a mixed-sex group of young adults, compared to young men living with an adult age 35+ (probably a parent). In contrast, women 18-24 living with other young women are just as likely to return all their diaries as those women living with an older adult.

The findings of this study have application in consideration of household composition when trying to gain survey cooperation. Whether targeting a single individual within the household or multiple household members, the dynamics of the household interaction can act to facilitate or put up barriers to participation. By knowing the household membership and anticipating its effect on cooperation, survey effort can be directed accordingly to maximize response rate. 